\title{
Influence of tetrahedral order on ferromagnetic gel phases
}

\author{
Tilen Potisk ${ }^{1, \mathrm{a}}$, Harald Pleiner ${ }^{2, \mathrm{~b}}$, and Helmut R. Brand ${ }^{1}$ \\ 1 Department of Physics, University of Bayreuth, 95440 Bayreuth, Germany \\ 2 Max Planck Institute for Polymer Research, 55021 Mainz, Germany
}

Received 10 January 2019

Published online: 25 March 2019

(C) The Author(s) 2019. This article is published with open access at Springerlink.com

\begin{abstract}
We investigate the macroscopic dynamics of gels with tetrahedral/octupolar symmetry, which possess in addition a spontaneous permanent magnetization. We derive the corresponding static and dynamic macroscopic equations for a phase, where the magnetization is parallel to one of the improper fourfold tetrahedral symmetry axes. Apart from elastic strains, we take into account relative rotations between the magnetization and the elastic network. The influence of tetrahedral order on these degrees of freedom is investigated and some experiments are proposed that are specific for such a material and allow to indirectly detect tetrahedral order. We also consider the case of a transient network and predict that stationary elastic shear stresses arise when a temperature gradient is applied.
\end{abstract}

\section{Introduction}

Ferrofluids are a well-established subfield of complex fluids $[1,2]$ showing a strong response to small external magnetic fields since they contain magnetic monodomain particles in a solvent. They have numerous applications including seals, dampers, loud speakers etc. They are strictly speaking super-paramagnetic and have no spontaneous magnetization in contrast to, for example, ferromagnetic solids. Their description on many length and time scales is well established [3-8]. Truly ferromagnetic fluids have not yet been reported for room temperature and ambient conditions.

More recently isotropic magnetic gels have been investigated for a number of polymeric systems containing magnetic particles and combining the properties of a ferrofluid with those of a polymeric gel [9-13]. Also their macroscopic and mesoscopic properties have been analyzed in some detail [14-16]. Synthesizing ferrogels in the presence of an external magnetic field has led to uniaxial magnetic gels $[10,17]$ with a finite magnetization $M_{0}$ in the absence of a magnetic field, which is implemented during the cross-linking process. This property is of high physical significance, since such systems show a linear response in a magnetic field [17], in addition to the usual quadratic field response. For both, isotropic ferrogels [14] and uniaxial [18] ferromagnetic gels, dynamic macroscopic descriptions are available.

We derive the hydrodynamics of tetrahedral ferromagnetic gels. They can be viewed as (uniaxial) magnetic gels

\footnotetext{
a e-mail: tilen.potisk@uni-bayreuth.de

b e-mail: pleiner@mpip-mainz.mpg.de
}

with an additional tetrahedral order. Uniaxial magnetic gels have been described theoretically in ref. [18] and are investigated experimentally in ref. [17]. Here we are interested in finding new effects that would hallmark the presence of an additional tetrahedral order. We consider predominantly the case of permanent gels, where elastic strains do not relax. As before, we will assume that the magnetic preferred direction is along one of the $\overline{4}$ axes of the tetrahedral order and that this coupling is rigid.

Here we investigate macroscopically the influence of octupolar/tetrahedral order on the physical properties of ferromagnetic gels. This issue is important, because the presence of octupolar order is associated with spontaneously broken inversion (parity) symmetry and can thus lead to static and dynamic cross-coupling effects absent otherwise. For gelled magnetic systems we are not aware of any previous study in this direction.

The major part of the investigations on the influence of octupolar order for fluid and gel-like systems has been in the field of liquid crystals [19-33] starting with the pioneering papers of Fel $[19,20]$. From an experimental point of view the systems of interest have been liquidcrystalline phases derived from bent-core or ferrocene-type molecules [34-48]. For a recent review of the field of tetrahedral order in liquid crystals we refer to ref. [49]. Quite recently, the hydrodynamics of tetrahedral ferromagnetic nematic fluids has been discussed [50]. Only rather recently the presence of octupolar order has been suggested as an explanation [51] for the observation of macroscopic chiral domains of either hand in some optically isotropic phases [52-54]. In ref. [51] it has been shown in the framework of macroscopic dynamics that the simultane- 
ous presence of octupolar order and a transient network is sufficient to produce ambidextrous helicity and thus to the possibility to obtain macroscopic chiral domains of either hand in compounds composed of non-chiral molecules.

The present paper is organized as follows. In sect. 2 we discuss the macroscopic dynamic description of ferromagnetic gel phases with octupolar order. This is followed in sect. 3 by suggestions of possible static and dynamic experiments that are specific for such phases including elasticity, tetrahedral and magnetic order. In sect. 4 we consider the case of a transient network and propose an experiment to study its influence. A summary and perspective, sect. 5, concludes the main text. In appendix A we list the full expressions for the thermodynamic conjugate forces and in appendix B we present the explicit expressions for the dissipative parts of the currents.

\section{Ferromagnetic gel phases with tetrahedral order}

\subsection{Macroscopic variables}

To derive the macroscopic equations of a particular macroscopic system one must first identify the relevant macroscopic variables. In addition to the conserved variables characteristic of an isotropic fluid, the mass density $\rho$, the energy density $\varepsilon$, the density of linear momentum $\mathbf{g}$, and the concentration $c$, related to an additional mass conservation law in binary mixtures, tetrahedral phases are described by a fully symmetric third rank tensor $T_{i j k}=$ $T_{0} \sum_{\beta=1}^{4} n_{i}^{\beta} n_{j}^{\beta} n_{k}^{\beta}$ [19], where the vectors $\mathbf{n}^{\beta}(\beta=1,2,3,4)$ span a tetrahedron and the order parameter $T_{0}$ (or rather $\left.\tilde{T}_{0} \equiv 4 T_{0} / 3 \sqrt{3}\right)$ describes the strength of the tetrahedral order, which we take as constant. Tetrahedral order breaks spatial inversion symmetry, but does not imply polar order, nor chirality. It fully breaks (spontaneously) rotational symmetry of isotropic space and its three independent rotations are the symmetry or Goldstone variables [49].

In ferromagnetic systems the spontaneous magnetization, $\mathbf{M}$, describes the strength of magnetic order by the order parameter $M \equiv|\mathbf{M}|$, and its orientation by the unit vector $\mathbf{m}=\mathbf{M} / M$. The former is neither connected to a Goldstone mode, nor to a conservation law, and therefore does not give rise to a genuine hydrodynamic variable. Nevertheless, its relaxation time can be large enough to be relevant in the hydrodynamic regime, and we will keep $\delta M \equiv M-M_{0}$, with $M_{0}$ the equilibrium magnetization, as a macroscopic variable.

The orientation of the magnetization breaks rotational symmetry of isotropic space partially twice, but is arbitrary in the absence of any orienting external field or boundary, constituting two Goldstone or symmetry variables $\delta \mathbf{m}$ with $\mathbf{m} \cdot \delta \mathbf{m}=0$. In this respect they are equivalent to director rotations in uniaxial nematic liquid crystals. However, in the present system, where rotational symmetry is already broken by the tetrahedral order, magnetic orientation does not give rise to additional independent Goldstone modes, and only the (three) combined (rigid) rotations of tetrahedral and magnetic orientations give rise to hydrodynamic degrees of freedom. Obviously, there must be an energetic penalty for rotations of the magnetization relative to the tetrahedral orientation.

Indeed, a simple Landau free energy argument shows that there are two energetic minima (stable thermodynamic phases), either with the magnetization pointing along one of the tetrahedral vectors, or along one of the improper $\overline{4}$ axes of the tetrahedron. The former is a phase of $C_{3 v}$ symmetry, which we will not consider here. The latter is a $D_{2 d}$ symmetric phase, similar to the tetrahedral nematic liquid crystal phase $D_{2 d}$ [49]. Nevertheless, the hydrodynamics of the ferromagnetic tetrahedral gel phase (called $D_{2 d}^{m g}$ in the following) is quite different from that of a $D_{2 d}$ phase, since the magnetization is a variable that changes sign under time reversal (in contrast to the nematic director) and since the $D_{2 d}$ phase is fluid. The static and the dynamic behavior of the $D_{2 d}^{m g}$ phase is also quite different from that of a tetrahedral ferromagnetic nematic phase [50], where the magnetization and the director field point both along one of the improper $\overline{4}$ axes. This phase includes as an example a dynamic interaction of the magnetization and the director field, which is different from the interplay of the magnetization and the strain field (defined at the end of this subsection). Furthermore, in a $D_{2 d}^{m g}$ phase one can induce an inhomogeneous rotation of the magnetization by application of an external strain, which, of course, has no effect in a tetrahedral ferromagnetic nematic phase, since the latter is fluid.

As hydrodynamic variables one can use the (three) combined rigid rotations of the tetrahedral structure together with the magnetization. An alternative possibility, more appropriate to the magnetic nature of the phase (and the application of external magnetic fields) is the use of the two magnetization rotations $\delta \mathbf{m}$ (implying an appropriate co-rotation of the tetrahedral structure to preserve rigidly the combined structure) and a rotation of the tetrahedral structure about the magnetization, $\delta \Omega$. The latter is not just a scalar variable, but has rather unusual rotational properties $[32,49]$.

We describe the elastic properties of a gel by the linearized version of the strain field $\varepsilon_{i j}=\frac{1}{2}\left(\nabla_{i} u_{j}+\nabla_{j} u_{i}\right)$ with $u_{i}$ the displacement field of the network. Note that $\varepsilon_{i j}$ is invariant under time reversal and spatial inversion. For a hydrodynamic implementation of nonlinear elasticity, see refs. $[55,56]$. We also consider as macroscopic variables the relative rotations between the preferred direction $m_{i}$ and the polymer network, defined linearly as $\tilde{O}_{i}=\delta m_{i}-\frac{1}{2} m_{j}\left(\nabla_{i} u_{j}-\nabla_{j} u_{i}\right)$. The relative rotations are transverse to the magnetization by construction $\left(m_{i} \tilde{O}_{i}=0\right)$, invariant under spatial inversion, but change sign under time reversal. For a nonlinear description of relative rotations we refer the reader to ref. [57]. From uniaxial nematic gels (with the director as preferred direction) it is well known that those relative rotations play a crucial role in the static and dynamic behavior of nematic gels $[58,59]$, and we expect similar importance for the magnetic case. 


\subsection{Thermodynamics and statics}

In this section we deal with static deviations from the equilibrium state. Changes of the variables introduced above are related to changes of the total energy density $f$ by the Gibbs relation, which is the local formulation of the first law of thermodynamics

$$
\begin{aligned}
\mathrm{d} f= & T \mathrm{~d} \sigma+\mu \mathrm{d} \rho+v_{i} \mathrm{~d} g_{i}+\mu_{c} \mathrm{~d} c+h^{M} \mathrm{~d} M \\
& +h_{i}^{m^{\prime}} \mathrm{d} m_{i}+\Psi_{i j}^{m} \mathrm{~d} \nabla_{j} m_{i}+h^{\Omega^{\prime}} \mathrm{d} \Omega+\Psi_{i}^{\Omega} \mathrm{d} \nabla_{i} \Omega \\
& +\Psi_{i j} \mathrm{~d} \varepsilon_{i j}+W_{i} \mathrm{~d} \tilde{O}_{i}
\end{aligned}
$$

where we have explicitly considered gradients of the rotational degrees of freedom, since they are symmetry variables and, in the absence of any orienting fields or boundaries, changes of their orientations must not change the energy $\left(h_{i}^{m^{\prime}}=0=h^{\Omega^{\prime}}\right)$. In the statics only the combinations

$$
h_{i}^{M}=h_{i}^{M^{\prime}}-\nabla_{j} \Psi_{i j}^{M} \quad \text { and } \quad h^{\Omega}=h^{\Omega^{\prime}}-\nabla_{i} \Psi_{i}^{\Omega}
$$

do occur.

The thermodynamic conjugates are the prefactors of the differentials in eq. (1), i.e. temperature $T$, chemical potential $\mu$, velocity $v_{i}$, osmotic pressure (divided by the pressure) $\mu_{c}$, so-called molecular fields of the magnetic order $h^{M}$, of the magnetization rotations $h_{i}^{m}$, of rotations about the magnetization $h^{\Omega}$ and the elastic stress, $\Psi_{i j}$ and the molecular field corresponding to the relative rotations, $W_{i}$. They (or their gradients) act as thermodynamic forces in the dynamics (depending whether they are zero or finite in equilibrium).

The energy has to be rotationally invariant. This requires for eq. (1) the condition

$$
\begin{aligned}
0= & \epsilon_{i j k}\left(h_{i}^{m^{\prime}} m_{j}+\Psi_{l i}^{m} \nabla_{j} m_{l}+\Psi_{i l}^{m} \nabla_{l} m_{j}+\Psi_{i}^{\Omega} \nabla_{j} \Omega\right. \\
& \left.+\Psi_{i l} \varepsilon_{j l}+\Psi_{l i} \varepsilon_{l j}+W_{i} \tilde{O}_{j}\right)-h^{\Omega} m_{k},
\end{aligned}
$$

which will later be used to symmetrize the stress tensor. The last term in this condition is due the fact that $\Omega$ is not a scalar, but a component of a vector that is not invariant under rotation. To find its rotational behaviour one can use the fact that $\delta T_{q k l}$ transforms under rotations like an ordinary vector in each of its indices [32].

Since the energy of the total volume, $F=\int f \mathrm{~d} V$, has to be a first order Eulerian form of the extensive variables, the thermodynamic pressure, $p \equiv-\partial F / \partial V$, can be written as a bilinear expression of the extensive variables and their thermodynamic conjugates with the final result (Gibbs-Duhem equation)

$$
\begin{aligned}
\mathrm{d} p= & \sigma \mathrm{d} T+\rho \mathrm{d} \mu+g_{i} \mathrm{~d} v_{i}+c \mathrm{~d} \mu_{c}+M \mathrm{~d} h^{M} \\
& -h_{i}^{m^{\prime}} \mathrm{d} m_{i}-\Psi_{i j}^{m} \mathrm{~d} \nabla_{j} m_{i}-h^{\Omega^{\prime}} \mathrm{d} \Omega-\Psi_{i}^{\Omega} \mathrm{d} \nabla_{i} \Omega \\
& -\varepsilon_{i j} \mathrm{~d} \Psi_{i j}-\tilde{O}_{i} \mathrm{~d} W_{i} .
\end{aligned}
$$

The thermodynamic conjugates are defined as partial derivatives of the total energy density with respect to the appropriate variable. Thus they follow from a total energy functional that can be written as

$$
f=f_{0}+f_{\text {rotel }}+f_{M}+f_{\text {lin }}+f_{\text {el }}+f_{\text {rr }},
$$

where $f_{0}$ is the total energy of an isotropic liquid, $f_{\text {rotel }}$ contains the rotational-elastic energy, $f_{M}$ is the magnetic energy including external magnetic fields, and $f_{\text {el }}$ contains the elastic energy and all cross-couplings of the strain tensor with the other variables (except relative rotations) and finally, $f_{\mathrm{rr}}$, shows all contributions of the relative rotations.

When constructing the explicit forms of the various energy contributions one can make use of the Levi-Civita tensor $\epsilon_{i j k}$, the tetrahedral structure $T_{i j k}$ and the magnetic direction $m_{i}$, where the latter two are not really independent. One has to note that $T_{i j k}$ is odd under spatial inversion and $m_{i}$ is odd under time reversal. In particular we find $[60]$

$$
\begin{aligned}
f_{0}= & \frac{T}{2 C_{V}}(\delta \sigma)^{2}+\frac{1}{2 \rho^{2} \kappa_{s}}(\delta \rho)^{2}+\frac{\gamma}{2}(\delta c)^{2}+\frac{1}{\rho \alpha_{s}}(\delta \sigma)(\delta \rho) \\
& +\beta_{\sigma}(\delta c)(\delta \sigma)+\beta_{\rho}(\delta c)(\delta \rho)+\frac{g_{i}^{2}}{2 \rho}
\end{aligned}
$$

containing the standard thermodynamic susceptibilities, like specific heat, compressibility, thermal expansion etc.

Rotations of the magnetization must not increase the total energy of the system, since $m_{i}$ is a symmetry variable. Therefore, only inhomogeneous rotations enter $f_{\text {rotel }}$

$$
\begin{aligned}
f_{\text {rotel }}= & \frac{1}{2} K_{i j k l}\left(\nabla_{j} m_{i}\right)\left(\nabla_{l} m_{k}\right)+\frac{1}{2} K_{i j}^{\Omega}\left(\nabla_{i} \Omega\right)\left(\nabla_{j} \Omega\right) \\
& +C_{i j k}^{m \Omega}\left(\nabla_{i} \Omega\right)\left(\nabla_{k} m_{j}\right)+\Pi_{i j k}^{c}\left(\nabla_{i} c\right)\left(\nabla_{k} m_{j}\right) \\
& +\Pi_{i j k}^{\sigma}\left(\nabla_{i} \sigma\right)\left(\nabla_{k} m_{j}\right)+\Pi_{i j k}^{\rho}\left(\nabla_{i} \rho\right)\left(\nabla_{k} m_{j}\right) \\
& +\left(\nabla_{i} \Omega\right)\left(C_{i j}^{c \Omega} \nabla_{j} c+C_{i j}^{\sigma \Omega} \nabla_{j} \sigma+C_{i j}^{\rho \Omega} \nabla_{j} \rho\right)
\end{aligned}
$$

with the rotational stiffness (or rotational elastic) tensors

$$
\begin{aligned}
K_{i j k l}= & K_{1} \delta_{i j}^{\perp} \delta_{k l}^{\perp}+K_{2} m_{p} m_{q} \epsilon_{i j p} \epsilon_{k l q} \\
& +K_{3} m_{j} m_{l} \delta_{i k}^{\perp}+K_{4} m_{p} m_{q} T_{i k p} T_{j l q}, \\
K_{i j}^{\Omega}= & K_{\perp}^{\Omega} \delta_{i j}^{\perp}+K_{\|}^{\Omega} m_{i} m_{j}, \\
C_{i j k}^{m \Omega}= & C_{\perp}\left(\epsilon_{j k p} m_{i}+\epsilon_{j i p} m_{k}\right) m_{p}, \\
\Pi_{i j k}^{\lambda}= & \Pi^{\lambda}\left(m_{i} \delta_{j k}^{\perp}+m_{k} \delta_{i j}^{\perp}\right), \\
C_{i j}^{\lambda \Omega}= & C_{\perp}^{\lambda} m_{k} T_{k p s}\left(\epsilon_{i r s} T_{j p r}+\epsilon_{j r s} T_{i p r}\right),
\end{aligned}
$$

where $\lambda \in\{\sigma, \rho, c\}$. The structure of $f_{\mathrm{el}}$ is isomorphic to the gradient energy in the $D_{2 d}$ phase and contains 4 coefficients related to bending distortions of the magnetization, 2 related to inhomogeneous rotations about the magnetization and 1 mixed one. In addition there are crosscouplings of those inhomogeneous rotations with gradients of the scalar conserved variables. We note that the contribution $\sim C_{\perp}$ is associated with $\nabla \times \mathbf{m}$; while this quantity typically vanishes statically, this need not be the case dynamically ${ }^{1}$.

1 The appropriate term $\sim K_{7}$ in refs. [49] and [32] should have the form of eq. (11) 
The magnetic part of the free energy in eq. (5) reads

$$
f_{M}=-M_{i} H_{i}+\frac{1}{2} \alpha M^{2}+\frac{1}{4} \beta M^{4}+f_{g M},
$$

where $f_{g M}$ contains gradients of the magnetic order parameter $M$, which are often neglected, but come here in parallel to gradients of the magnetic direction $m_{j}$. The homogeneous part of $f_{M}$ is derived in sect. 2.4 taking into account the static magnetic Maxwell equations. $f_{M}$ is the Legendre transformed magnetic energy containing the magnetic field $\mathbf{H}$. The ferromagnetic coupling in $f_{M}$ leads to the parallel equilibrium orientation of the magnetization along an external magnetic field. As a result a homogeneous external field is compatible with a homogeneous combined magnetization/tetrahedral structure in the $D_{2 d}^{m}$ phase. However, the degeneracy of the (combined) orientation of the magnetization and the tetrahedral structure is partially lifted and only the orientation of the structure perpendicular to the field (and $\mathbf{m}$ ) is still arbitrary. A discussion on how the degeneracy can completely be eliminated by additional (e.g., electric fields) is discussed below.

For the magnetic gradient energy we find

$$
\begin{aligned}
f_{g M}= & \frac{1}{2} K_{i j}^{M}\left(\nabla_{i} M\right)\left(\nabla_{j} M\right)+C_{i j}^{M \Omega}\left(\nabla_{i} M\right)\left(\nabla_{j} \Omega\right) \\
& +K_{i j k}^{M m}\left(\nabla_{i} M\right)\left(\nabla_{j} m_{k}\right) \\
& +\left(\nabla_{i} M\right)\left(\Pi_{i j}^{c M} \nabla_{j} c+\Pi_{i j}^{\sigma M} \nabla_{j} \sigma+\Pi_{i j}^{\rho M} \nabla_{j} \rho\right)
\end{aligned}
$$

with

$$
\begin{aligned}
K_{i j}^{M} & =K_{\perp}^{M} \delta_{i j}^{\perp}+K_{\|}^{M} m_{i} m_{j}, \\
C_{i j}^{M \Omega} & =C_{\|} m_{k} T_{k p s}\left(\epsilon_{i r s} T_{j p r}+\epsilon_{j r s} T_{i p r}\right), \\
K_{i j k}^{M m} & =K^{M m}\left(m_{j} \delta_{i k}^{\perp}+m_{i} \delta_{j k}^{\perp}\right), \\
\Pi_{i j}^{\lambda M} & =\Pi_{\perp}^{\lambda M} \delta_{i j}^{\perp}+\Pi_{\|}^{\lambda M} m_{i} m_{j},
\end{aligned}
$$

where $\lambda \in\{\sigma, \rho, c\}$. There are two stiffness coefficients $\left(K_{\perp}^{M}, K_{\|}^{M}\right)$ related to distortions of $M$. Cross-couplings between distortions of $M$ and inhomogeneous rotations of, and about the magnetization, are described by one coefficient each ( $K^{M m}$ and $C_{\|}$, respectively), while there are in total six coefficients $\left(\Pi_{\perp, \|}^{\lambda M}\right)$ connected to the coupling of gradients of $M$ with gradients of the scalar conserved variables.

The next energy contribution we are discussing here is the linear gradient energy

$$
f_{\text {lin }}=\xi T_{i j k} m_{i}\left(\nabla_{j} m_{k}\right) .
$$

This expression is identical to the linear gradient term in the $D_{2 d}$ phase [32], when $m_{i}$ is replaced by the director $n_{i}$. This linear gradient term is allowed due to the presence of tetrahedral order, which breaks parity. As a consequence, the ground state might not be homogeneous, resembling the case of added chirality to nematic liquid crystals. However, here is no chirality involved and helices of both rotation sense are equally well possible (ambidextrous chirality) [49]. This is further investigated in appendix A.
The elastic part of the energy reads

$$
\begin{aligned}
f_{\mathrm{el}}= & \frac{1}{2} c_{i j k l} \varepsilon_{i j} \varepsilon_{k l}-\gamma_{i j} \varepsilon_{i j} M_{0} \delta M \\
& +\left(\chi_{i j}^{\sigma} \delta \sigma+\chi_{i j}^{\rho} \delta \rho+\chi_{i j}^{c} \delta c\right) \varepsilon_{i j} \\
& +\left(\tau_{i j k}^{\sigma} \nabla_{k} \sigma+\tau_{i j k}^{c} \nabla_{k} c+\tau_{i j k}^{\rho} \nabla_{k} \rho+\tau_{i j k}^{M} \nabla_{k} M\right) \varepsilon_{i j} \\
& +\chi_{i j k}^{\Omega} \varepsilon_{i j} \nabla_{k} \Omega+\chi_{i j k l}^{m} \varepsilon_{i j} \nabla_{l} m_{k} .
\end{aligned}
$$

The first two lines of $f_{\text {el }}$ are known from uniaxial magnetic gels [18]. The elastic tensor $c_{i j k l}$ is of the form of the viscosity tensor, eq. (46), and has six elastic moduli, $c_{1, \ldots, 6}$, one more than in uniaxial magnetic gels due to the tetrahedral order. The rank-2 tensors are of the standard uniaxial form, eq. (15), and describe magnetostriction as well as elastic deformations due to changes in temperature, density or concentration. The third line describes static couplings between gradients of temperature, density, concentration or magnitude of the magnetization with elastic deformations with the tensors

$$
\tau_{i j k}^{\lambda}=\left(\tau_{\perp}^{\lambda} \delta_{k l}^{\perp}+\tau_{\|}^{\lambda} m_{k} m_{l}\right) T_{i j l}
$$

containing two coefficients for each $\lambda \in\{\sigma, \rho, c, M\}$. In ref. [51] such a coupling is also present, but shows only one coefficient for each $\lambda$ due to the optically isotropic nature of the $T_{d}$ phase. The fourth line contains couplings genuine for the $D_{2 d}^{m g}$ phase between elastic deformations and inhomogeneous rotations of the tetrahedral/magnetization structure with the material tensors

$$
\begin{aligned}
\chi_{i j k}^{\Omega} & =\chi_{1}^{\Omega}\left(\epsilon_{i p r} T_{j k p}+\epsilon_{j p r} T_{i k p}\right) m_{r}+\chi_{2}^{\Omega} \epsilon_{k p r} T_{i j p} m_{r}, \\
\chi_{i j k l}^{m} & =\delta_{k p}^{\perp}\left(\chi_{1}^{m}\left[T_{i p l} m_{j}+T_{j p l} m_{i}\right]+\chi_{2}^{m} T_{i j p} m_{l}\right) .
\end{aligned}
$$

Finally, the energy containing relative rotations

$$
\begin{aligned}
f_{\mathrm{rr}}= & \frac{1}{2} D_{1} \tilde{O}_{i} \tilde{O}_{i}+D_{2}\left(m_{j} \delta_{i k}^{\perp}+m_{k} \delta_{i j}^{\perp}\right) \tilde{O}_{i} \varepsilon_{j k} \\
& +\left(\psi_{i j}^{c} \nabla_{i} c+\psi_{i j}^{\rho} \nabla_{i} \rho+\psi_{i j}^{\sigma} \nabla_{i} \sigma+\psi_{i j}^{M} \nabla_{i} M\right) \tilde{O}_{j}
\end{aligned}
$$

contains the stiffness of relative rotations, $D_{1}$, and the standard uniaxial coupling between elasticity and relative rotations, $D_{2}$, well known from nematic and magnetic gels. The second line describes the genuine couplings of gradients of temperature, density, concentration, and magnitude of the magnetization with relative rotations in the $D_{2 d}^{m g}$ phase by

$$
\psi_{i j}^{\lambda}=\psi^{\lambda} m_{k} T_{i j k}
$$

The expressions for the thermodynamic conjugates that follow from the energy contributions introduced above are listed in appendix A.

\subsection{Dynamic equations}

The hydrodynamic variables can be put into two different classes. There are conserved variables, like the mass density, energy density and momentum density $\mathbf{g}$, which are 
governed by conservation laws. The second class of variables corresponds to the variables associated with spontaneously broken continuous symmetries. Their dynamics is governed by balance laws. In our case we have from this class the magnetization and the rotation around the magnetization $\Omega$. There are some variables, that relax on a finite but very long time scale and it is therefore sensible to include them into the macroscopic description, ref. [60]. In our case we will consider the relative rotations $\tilde{O}_{i}$, which are important, if the magnetization and the strain are weakly coupled.

The dynamic equations read

$$
\begin{aligned}
\frac{\partial}{\partial t} f+\nabla_{i}\left([f+p] v_{i}+j_{i}^{f}\right) & =0, \\
\frac{\partial}{\partial t} \rho+\nabla_{i} g_{i} & =0, \\
\frac{\partial}{\partial t} g_{i}+\nabla_{j}\left(g_{i} v_{j}+p \delta_{i j}+\sigma_{i j}^{t h}+\sigma_{i j}\right) & =0, \\
\frac{\partial}{\partial t} \sigma+\nabla_{i}\left(\sigma v_{i}+j_{i}^{\sigma}\right) & =\frac{2 R}{T}, \\
\rho\left(\frac{\partial}{\partial t}+v_{j} \nabla_{j}\right) c+\nabla_{i} j_{i}^{c} & =0, \\
\left(\frac{\partial}{\partial t}+v_{j} \nabla_{j}\right) M+X^{M} & =0, \\
\left(\frac{\partial}{\partial t}+v_{j} \nabla_{j}\right) m_{i}-\epsilon_{i j k} \omega_{j} m_{k}+X_{i}^{m} & =0, \\
\left(\frac{\partial}{\partial t}+v_{j} \nabla_{j}\right) \Omega-m_{i} \omega_{i}+Z & =0, \\
\left(\frac{\partial}{\partial t}+v_{k} \nabla_{k}\right) \varepsilon_{i j}+Y_{i j}^{t h}+Y_{i j} & =0 \\
\left(\frac{\partial}{\partial t}+v_{j} \nabla_{j}\right) \tilde{O}_{i}+\epsilon_{i j k} \tilde{O}_{j} \omega_{k}+Y_{i}^{O} & =0
\end{aligned}
$$

with the vorticity $\omega_{i}=(1 / 2) \epsilon_{i j k} \nabla_{j} v_{k}$. The vorticity contributions are due to the fact that $m_{i}$ and $\tilde{O}_{i}$ transform under spatial rotations as vectors, and $\Omega$ as a special component of a vector [49]. These terms ensures that only those rotations enter hydrodynamics that go beyond the global rotation (e.g., of the coordinate system). In eq. (33) the $m_{i} \omega_{i}$ term shows again that $\Omega$ is not a scalar quantity.

In eq. (34) we have introduced the nonphenomenological current of the strain as

$$
Y_{i j}^{t h}=-A_{i j}+\varepsilon_{k j} \nabla_{i} v_{k}+\varepsilon_{k i} \nabla_{j} v_{k}
$$

containing $A_{i j}=(1 / 2)\left(\nabla_{i} v_{j}+\nabla_{j} v_{i}\right)$ due to the translational nature of the displacement field, as well as the corotational part of the time derivative of the strain tensor.

In eq. (28) we have explicitly written down the nonphenomenological part of the stress tensor $\nabla_{i} p$, the pressure gradient given by eq. (4), and $\sigma_{i j}^{\text {th }}$ given by

$$
\begin{aligned}
2 \sigma_{i j}^{t h}= & \Psi_{k j}^{m} \nabla_{i} m_{k}+\Psi_{k i}^{m} \nabla_{j} m_{k}+\Psi_{j}^{\Omega} \nabla_{i} \Omega+\Psi_{i}^{\Omega} \nabla_{j} \Omega \\
& +\nabla_{k}\left(m_{j} \Psi_{i k}^{m}-m_{i} \Psi_{j k}^{m}\right)-2 \Psi_{i j}+\Psi_{i k} \varepsilon_{k j}+\Psi_{j k} \varepsilon_{k i}
\end{aligned}
$$

which has been brought, using eq. (3), into the form [61] that guarantees angular momentum conservation [62]. The three last terms describe linear and nonlinear elastic stresses.

The source term in the dynamic evolution equation for the entropy density, eq. (29), is proportional to the dissipation function $R$ representing (half of) the rate at which the heat is transferred to the microscopic degrees of freedom. The second law of thermodynamics requires $R>0$ for dissipative processes, while $R=0$ holds for the reversible parts of the currents, in which case eq. (29) is a conservation law. Splitting the phenomenological currents $\left(j_{i}^{f}, \sigma_{i j}, j_{i}^{\sigma}, j_{i}^{c}, X^{M}, X_{i}^{m}, Z, Y_{i j}, Y_{i}^{O}\right)$ into the dissipative part (superscript $D$ ) and the reversible one (superscript $R$ ) the Gibbs relation eq. (1) then leads to the condition

$$
\begin{aligned}
2 R= & -\nabla_{i} j_{i}^{f D}-j_{i}^{\sigma D} \nabla_{i} T-j_{i}^{c D} \nabla_{i} \mu_{c}-\sigma_{i j}^{D} A_{i j}+X_{i}^{m D} h_{i}^{m} \\
& +X^{M D} h^{M}+Z^{D} h^{\Omega}+Y_{i j}^{D} \Psi_{i j}+Y_{i}^{O D} W_{i}>0
\end{aligned}
$$

for dissipative processes, where only the symmetrized velocity gradient $A_{i j}$ enters, in order to prevent solid body rotations to produce entropy.

For reversible currents, the condition

$$
\begin{aligned}
0= & -\nabla_{i} j_{i}^{f R}-j_{i}^{\sigma R} \nabla_{i} T-j_{i}^{c R} \nabla_{i} \mu_{c}-\sigma_{i j}^{R} A_{i j}+X^{M R} h^{M} \\
& +X_{i}^{m R} h_{i}^{m}+Z^{R} h^{\Omega}+Y_{i j}^{R} \Psi_{i j}+Y_{i}^{O R} W_{i}
\end{aligned}
$$

applies. Possible pure divergence contributions (surface terms) are put into $j_{i}^{f}$, but are not needed in the following. The various transport contributions in the time derivatives of eqs. (26)-(35) are all reversible. Their zero entropy production is ensured by the non-phenomenological parts of the stress tensor $\sigma_{i j}^{t h}$ and by the pressure $p$. Similarly, $Y_{i j}^{t h}$ compensates the linear and nonlinear elastic stresses in $\sigma_{i j}^{\text {th }}$ to give $R=0$.

A current is reversible, if it transforms under time reversal in the same way as the time derivative of the appropriate variable, while the dissipative part of a current has the opposite time reversal behavior. In the following we will discuss the dissipative and reversible dynamics separately.

To derive the dissipative parts of the phenomenological currents one first writes the dissipation function as a positive quadratic form in the thermodynamic forces taking into account that $R$ has to be a time reversal symmetric, scalar quantity. By taking the variational derivative of this function with respect to the chosen thermodynamic force one gets the corresponding dissipative current. The dissipation function reads

$$
\begin{aligned}
R= & \frac{1}{2} \kappa_{i j}\left(\nabla_{i} T\right)\left(\nabla_{j} T\right)+\frac{1}{2} D_{i j}\left(\nabla_{i} \mu_{c}\right)\left(\nabla_{j} \mu_{c}\right) \\
& +D_{i j}^{T}\left(\nabla_{i} T\right)\left(\nabla_{j} \mu_{c}\right)+\Gamma_{i j k}^{(2)} A_{i j} \nabla_{k} T+\Gamma_{i j k}^{(3)} A_{i j} \nabla_{k} \mu_{c} \\
& +\frac{1}{2} \nu_{i j k l}^{D} A_{i j} A_{k l}+c_{i j k}^{D} A_{i j} h_{k}^{m}+c_{i j}^{M} A_{i j} h^{M} \\
& +\tau_{i j} A_{i j} h^{\Omega}+T_{i j k} m_{j}\left(\psi^{T D} \nabla_{k} T+\psi^{c D} \nabla_{k} \mu\right) h_{i}^{m}
\end{aligned}
$$




$$
\begin{aligned}
& +\frac{1}{2} b_{i j}^{D} h_{i}^{m} h_{j}^{m}+\frac{1}{2} b^{M} h^{M} h^{M}+\frac{1}{2} b^{\Omega} h^{\Omega} h^{\Omega} \\
& +\frac{1}{2} D_{i j}^{\Psi}\left(\nabla_{k} \Psi_{i k}\right)\left(\nabla_{l} \Psi_{j l}\right)+\frac{1}{2} \tau^{W} W_{i} W_{i} \\
& +\left(\xi_{i j}^{T} \nabla_{i} T+\xi_{i j}^{c} \nabla_{i} \mu_{c}+\xi_{i j}^{M} \nabla_{i} h^{M}\right)\left(\nabla_{k} \Psi_{j k}\right) \\
& +\xi_{i j k}^{A} A_{i j} W_{k}+T_{i j k} m_{k}\left(p^{W} W_{i}+p^{m} h_{i}^{m}\right) \nabla_{l} \Psi_{j l} \\
& +T_{i j k} m_{k}\left(p^{T} \nabla_{j} T+p^{c} \nabla_{j} \mu_{c}+p^{M} \nabla_{j} h^{M}\right) W_{i} \\
& +f_{i j k}^{D} A_{i j} \nabla_{l} \Psi_{k l}+\xi^{W m} \delta_{i j}^{\perp} W_{i} h_{j}^{m} .
\end{aligned}
$$

Note that we have used the divergence of the elastic stress, $\nabla_{j} \Psi_{i j}$, as the thermodynamic force, rather than the elastic stress tensor itself, $\Psi_{i j}$. Using the latter there are additional contributions to the dissipation function, which will be discussed in detail in sect. 4 on transient networks. Since we assume in this section that the network of the gel is permanent, elastic strains can only diffuse, but not relax. Therefore we discard the part of the dissipation function associated with transient networks, eq. (87), and write in the following the elastic currents in the form

$$
Y_{i j}^{D, R}=-\frac{1}{2}\left(\nabla_{j} F_{i}^{D, R}+\nabla_{i} F_{j}^{D, R}\right)
$$

which reflects the definition of the linear strain tensor. in terms of the displacement vector. The dissipative expression $F_{i}^{D}$ follows from eq. (40) by $F_{i}^{D}=\partial R /\left(\partial \nabla_{k} \Psi_{i k}\right)$.

The dissipative currents for permanent networks will be given explicitly in appendix $\mathrm{B}$.

The dissipative material tensors $\kappa_{i j}, D_{i j}, D_{i j}^{T}, \tau_{i j}, D_{i j}^{\Psi}$, $\xi_{i j}^{T}, \xi_{i j}^{c}$ and $\xi_{i j}^{M}$ are of the standard uniaxial form

$$
\zeta_{i j}^{D}=\zeta_{1}^{D} \delta_{i j}^{\perp}+\zeta_{2}^{D} m_{i} m_{j}
$$

with a perpendicular and a parallel component, while the others read

$$
\begin{aligned}
\Gamma_{i j k}^{(2)}= & \Gamma_{21}^{D} \epsilon_{k p r} T_{i j p} m_{r} \\
& +\Gamma_{22}^{D}\left(\epsilon_{i p r} T_{k j p} m_{r}+\epsilon_{j p r} T_{k i p} m_{r}\right) \\
\Gamma_{i j k}^{(3)}= & \Gamma_{31}^{D} \epsilon_{k p r} T_{i j p} m_{r} \\
& +\Gamma_{32}^{D}\left(\epsilon_{i p r} T_{k j p} m_{r}+\epsilon_{j p r} T_{k i p} m_{r}\right), \\
f_{i j k}^{D}= & f_{1}^{D} \epsilon_{k p r} T_{i j p} m_{r} \\
& +f_{2}^{D}\left(\epsilon_{i p r} T_{k j p} m_{r}+\epsilon_{j p r} T_{k i p} m_{r}\right) \\
\nu_{i j k l}^{D}= & \nu_{1} \delta_{i j}^{\perp} \delta_{k l}^{\perp}+\nu_{2}\left(\delta_{j l}^{\perp} \delta_{i k}^{\perp}+\delta_{i l}^{\perp} \delta_{j k}^{\perp}\right) \\
& +\nu_{3} m_{i} m_{j} m_{k} m_{l}+\nu_{4}\left(\delta_{i j}^{\perp} m_{k} m_{l}+\delta_{k l}^{\perp} m_{i} m_{j}\right) \\
& +\nu_{5}\left(\delta_{i k}^{\perp} m_{j} m_{l}+\delta_{j k}^{\perp} m_{i} m_{l}+\delta_{i l}^{\perp} m_{j} m_{k}+\delta_{j l}^{\perp} m_{i} m_{k}\right) \\
& +\nu_{6} m_{p} m_{q} T_{i j p} T_{k l q}, \\
c_{i j k}^{D}= & c_{1}^{D}\left(\epsilon_{i k p} m_{j}+\epsilon_{j k p} m_{i}\right) m_{p}, \\
\xi_{i j k}^{A}= & \xi^{A}\left(\epsilon_{i k p} m_{j}+\epsilon_{j k p} m_{i}\right) m_{p}, \\
c_{i j}^{M}= & c_{2}^{D}\left(\epsilon_{i r s} T_{j p r}+\epsilon_{j r s} T_{i p r}\right) m_{k} T_{k p s} .
\end{aligned}
$$

The reversible parts of the currents do not follow from any potential, but can be derived by requiring that the entropy production $R$ in eq. (38) is zero. Replacing there $Y_{i j}^{R} \Psi_{i j}$ by $F_{i}^{R} \nabla_{k} \Psi_{i k}$ one gets

$$
\begin{aligned}
j_{i}^{\sigma R}= & -\kappa_{i j}^{R} \nabla_{j} T-D_{i j}^{T R} \nabla_{j} \mu_{c}+\psi_{i j}^{T} h_{j}^{m}+\Gamma_{k j i}^{T} A_{j k} \\
& -d_{i j}^{T} W_{j}-f^{T} \epsilon_{i j k} m_{j} \nabla_{l} \Psi_{k l} \\
j_{i}^{c R}= & -D_{i j}^{R} \nabla_{j} \mu_{c}+D_{i j}^{T R} \nabla_{j} T+\psi_{i j}^{c} h_{j}^{m}+\Gamma_{k j i}^{c} A_{j k} \\
& -d_{i j}^{c} W_{j}-f^{c} \epsilon_{i j k} m_{j} \nabla_{l} \Psi_{k l} \\
\sigma_{i j}^{R}= & -\nu_{i j k l}^{R} A_{k l}-c_{k i j}^{R} h_{k}^{m}-c_{i j}^{R} h^{M}-\tau_{i j}^{R} h^{\Omega} \\
& +d^{A}\left(m_{i} \delta_{k j}^{\perp}+m_{j} \delta_{k i}^{\perp}\right) W_{k}+f_{k j i}^{A} \nabla_{l} \Psi_{k l} \\
& -\Gamma_{i j k}^{T} \nabla_{k} T-\Gamma_{i j k}^{c} \nabla_{k} \mu_{c} \\
X_{i}^{m R}= & b_{i j}^{R} h_{j}^{m}-c_{i j k}^{R} A_{j k}+\psi_{j i}^{T} \nabla_{j} T+\psi_{j i}^{c} \nabla_{j} \mu_{c} \\
& +d^{m} \epsilon_{i j k} m_{j} W_{k}, \\
X^{M R}= & -c_{i j}^{R} A_{i j}, \\
Z^{R}= & -\tau_{i j}^{R} A_{i j}+f_{i j}^{\Omega} \nabla_{j} \nabla_{k} \Psi_{i k} \\
F_{i}^{R}= & d_{k i}^{\Psi} W_{k}+f^{T} \epsilon_{i j k} m_{j} \nabla_{k} T+f^{c} \epsilon_{i j k} m_{j} \nabla_{k} \mu_{c} \\
& +f^{\Psi} \epsilon_{i j k} m_{j} \nabla_{l} \Psi_{k l}+f_{i j}^{\Omega} \nabla_{j} h^{\Omega}+f_{i j k}^{A} A_{j k} \\
Y_{i}^{O R}= & d_{i j}^{T} \nabla_{j} T+d_{i j}^{c} \nabla_{j} \mu_{c}+d_{i j}^{\Psi} \nabla_{k} \Psi_{j k}+d^{W} \epsilon_{i j k} m_{j} W_{k} \\
& +d^{m} \epsilon_{i j k} m_{j} h_{k}^{m}+d^{A}\left(m_{k} \delta_{i l}^{\perp}+m_{l} \delta_{i k}^{\perp}\right) A_{k l}
\end{aligned}
$$

where the tensors $f_{i j}^{\Omega}$ and $c_{i j}^{R}$ are of the standard uniaxial form, eq. (15) with two coefficients $f_{\perp, \|}^{\Omega}$ and $c_{\perp, \|}^{R}$, respectively. The antisymmetric tensors $\kappa_{i j}^{R}, D_{i j}^{T R}, D_{i j}^{R}$, and $b_{i j}^{R}$ have only one coefficients and are of the form

$$
\kappa_{i j}^{R}=\kappa^{R} \epsilon_{i j k} m_{k},
$$

while for the other tensors we find

$$
\begin{aligned}
\psi_{i j}^{c, T}= & \psi^{c, T} \epsilon_{i p r} T_{j p k} m_{k} m_{r} \\
d_{i j}^{T, c, \Psi}= & d^{T, c, \Psi} \epsilon_{i p r} T_{j p k} m_{k} m_{r}, \\
\nu_{i j k l}^{R}= & \nu_{1}^{R}\left(\epsilon_{i k p} \delta_{j l}^{\perp}+\epsilon_{j k p} \delta_{i l}^{\perp}+\epsilon_{i l p} \delta_{j k}^{\perp}+\epsilon_{j l p} \delta_{i k}^{\perp}\right) m_{p} \\
& +\nu_{2}^{R}\left(\epsilon_{i k p} m_{j} m_{l}+\epsilon_{j l p} m_{i} m_{k}+\epsilon_{i l p} m_{j} m_{k}\right. \\
& \left.+\epsilon_{j k p} m_{i} m_{l}\right) m_{p}+\nu_{3}^{R} T_{i j p} T_{k l r}, \epsilon_{p r s} m_{s}, \\
c_{i j k}^{R}= & c_{1}^{R}\left(m_{j} \delta_{i k}^{\perp}+m_{k} \delta_{i j}^{\perp}\right) \\
\tau_{i j}^{R}= & \tau^{R}\left(\epsilon_{i r s} T_{j p r}+\epsilon_{j r s} T_{i p r}\right) m_{k} T_{k p s}, \\
\Gamma_{k j i}^{T, c}= & T_{q j k}\left(\Gamma_{\perp}^{T, c} \delta_{q i}^{\perp}+\Gamma_{\|}^{T, c} m_{q} m_{i}\right) \\
f_{i j k}^{A}= & T_{q j k}\left(f_{\perp}^{A} \delta_{q i}^{\perp}+f_{\|}^{A} m_{q} m_{i}\right) .
\end{aligned}
$$

The dissipative coupling between flow and gradients of temperature and concentration, described by $\Gamma_{21}^{D}, \Gamma_{22}^{D}, \Gamma_{31}^{D}$ and $\Gamma_{32}^{D}$ in eqs. (43) and (44), is genuine for magnetic tetrahedral fluids, while their reversible counterparts, $\Gamma_{\perp}^{T, c}$ and $\Gamma_{\|}^{T, c}$ and in eqs. (50) and (51), are already known from the nematic tetrahedral $D_{2 d}$ phase, refs. [32,49], and for $\Gamma_{\perp}^{T, c}=\Gamma_{\|}^{T, c}$ from the isotropic $T_{d}$ phase, refs. [27, 49]. Similarly, for the reversible cross-coupling between rotations of the magnetization and gradients of temperature and concentration, $\psi_{i j}^{c, T}$, eq. (59), are already found 
in $D_{2 d}$. Their dissipative counterparts, $\psi^{T D}$ and $\psi^{c D}$, eqs. (B.1), (B.2) and (B.4), are genuine for a tetrahedral ferromagnetic (fluid) phase. This is also true for the dissipative coupling between flow and changes of $M$, provided by $c_{i j}^{M}$, eq. (49), and the reversible coupling between flow and rotations about the magnetization, $\tau_{i j}^{R}$, eq. (63). Couplings provided by $f_{1}^{D}, f_{2}^{D}, p^{W}, p^{m}, p^{T}, p^{c}$ are genuine for the $D_{2 d}^{m g}$ phase and require the simultaneous presence of tetrahedral and magnetic order and of elasticity.

\subsection{External fields}

In the case that we have not only an external magnetic, but also an electric field, there are many competing orienting energy contributions

$$
\begin{aligned}
f_{\text {fields }}= & -M_{i} H_{i}+a T_{i l k} T_{j m k} M_{i} M_{l} M_{j} M_{m} \\
& -\zeta_{1} T_{i j k} E_{i} E_{j} E_{k}-\zeta_{2} T_{i j k} E_{i} H_{j} H_{k} \\
& -\zeta_{3} T_{i j k} E_{i} M_{j} H_{k}-\zeta_{4} T_{i j k} E_{i} M_{j} M_{k},
\end{aligned}
$$

which we discuss in the spirit of a Landau description. The first term, the ferromagnetic coupling between the magnetization and the external magnetic field, aligns the magnetization along the field and the second one governs the relative orientation of the magnetization with the tetrahedron, which for $a>0$, leads to the $D_{2 d}^{m g}$ structure. The terms $\sim \zeta_{1,2}$ are the typical couplings of external fields with the tetrahedral orientation, present in any tetrahedral phase. The last two terms $\sim \zeta_{3,4}$ are specific for the magnetic tetrahedral phase.

A full minimization of $f_{\text {fields }}$ is beyond the scope of this work. We look for some special cases. First, we assume that the energy contribution $\sim a$, which defines the structure of the $D_{2 d}^{m g}$ phase, is the dominant one $(a \rightarrow \infty)$. In that case the energy contribution $\sim \zeta_{4}$ is identically zero for all orientations of the fields. The ferromagnetic energy is minimal for the magnetization (and thus the $\overline{4}$ axis of the tetrahedron) to be parallel with the magnetic field (z-axis), while the cubic electric field contribution is minimal, if the electric field is parallel (for $\zeta_{1}>0$ ) or antiparallel (for $\zeta_{1}<0$ ) to one of the tetrahedral axes. However, the tetrahedral vectors make a finite angle $\theta_{T} / 2$ (with $\cos \left(\theta_{T} / 2\right)= \pm 1 / \sqrt{3}$ ) with the $\overline{4}$ axes, leading to frustration (except for the very special case that the two external fields make an angle of $\left.\theta_{T} / 2\right)$. Since the magnetic field does not fix the transverse structure, the energy $\sim \zeta_{1}$ can be minimized independently with regard to this transverse direction. As a result, the directions $\hat{\mathbf{e}}_{x} \pm \hat{\mathbf{e}}_{y}$ are given by (the tilt direction of) the electric field (the $\zeta_{2,3}$ energies do not change that statement).

The frustration of the orientation of the magnetization (or $\overline{4}$ axis) with respect to the orientation of the external fields, can be discussed along the lines of the liquid crystal case [32]. For strong magnetic and weak electric fields the ferromagnetic energy will win, while for weak magnetic and strong electric fields the magnetization will approach the orientation of the electric field. The transition process is governed by the parameters $\beta_{1,2,3}$, indicating the balance of ferromagnetic energy to those including electric fields, $\sim \zeta_{1,2,3}$, respectively

$$
\begin{aligned}
\beta_{1} & =\frac{M_{0} H_{0}}{\zeta_{1} T_{0} E_{0}^{3}}, \\
\beta_{2} & =\frac{M_{0}}{\zeta_{2} T_{0} E_{0} H_{0}}, \\
\beta_{3} & =\frac{1}{\zeta_{3} T_{0} E_{0}} .
\end{aligned}
$$

Qualitatively, a large $\beta_{1}$ leads to an orientation of the magnetization close to that of the magnetic field, while a small $\beta_{1}$ results in an orientation close to the electric field. The parameters $\beta_{2,3}$ come into play only for orientations in-between, neither very close to the magnetic, nor very close to the electric field.

If there is only an electric, but no magnetic field, the former fixes the orientation of the tetrahedron according to the $\zeta_{1}$ energy. In the case of the $D_{2 d}^{m g}$ phase, where the magnetization is along one of the $\overline{4}$ axes, however, the $\zeta_{1}$ energy vanishes and the electric field does not orient the tetrahedron. But the electric field induces elastic deformations according to the energy

$$
f_{\text {estrict }}=-\zeta_{\varepsilon} T_{i j k} E_{i} \varepsilon_{j k}
$$

that leads to elastic stresses

$$
\begin{aligned}
\Psi_{x y}^{e s} & =\zeta_{\varepsilon} \tilde{T}_{0} E_{\|}, \\
\sqrt{\left(\Psi_{x z}^{e s}\right)^{2}+\left(\Psi_{y z}^{e s}\right)^{2}} & =\zeta_{\varepsilon} \tilde{T}_{0} E_{\perp}
\end{aligned}
$$

for the field parallel to the magnetization $\left(E_{\|}\right)$and perpendicular to it $\left(E_{\perp}\right)$, respectively. The elastic stresses are compensated by appropriate deformations in the equilibrium state. This constitutes linear electrostriction in a magnetic phase that does not possess a permanent electric polarization.

On the other hand, there is a permanent magnetization and therefore magnetostriction

$$
f_{\text {mstrict }}=-\frac{1}{2} \gamma_{i j k l} M_{k} M_{l} \varepsilon_{i j}
$$

leading to elastic strains of a completely different form

$$
\begin{aligned}
& \Psi_{x x}^{m s}=\Psi_{y y}^{m s}=-\gamma_{\perp} M_{0}^{2}, \\
& \Psi_{z z}^{m s}=-\gamma_{\|} M_{0}^{2} .
\end{aligned}
$$

In equilibrium they are compensated by appropriate deformations, and hydrodynamic deviations are described by the energy $\sim \gamma_{i j}$ in eq. (20), with $\gamma_{i j}=\gamma_{i j z z}$. In the case of an additional external magnetic field (but without an electric one) $M_{0}$ is replaced by $M_{0}+H_{0}$. In the presence of both external fields the strictive deformations are extremely complicated due to the complicated orientation of the tetrahedron.

From eqs. (71) and (73) we read off immediately that external electric and magnetic fields lead to an anisotropy 
of the stresses and thus also of the associated strains. This anisotropy, which is probably very small, is neglected. We note that the coefficient $\alpha$ in eq. (13) is also modified by external fields due to the effects of the equilibrium strains $\varepsilon_{i j}^{0}$. The corresponding anisotropy will be neglected as well.

\section{Suggestions for experiments involving elasticity}

In this section we discuss experiments that are specific for the ferromagnetic tetrahedral gel phases. In particular, we consider experiments in the homogeneous state with a constant orientation of the preferred direction. We propose experiments that probe static couplings specific for the presence of tetrahedral order in sect. 3.1. In particular, we consider static external strains applied to the gel that affect the magnitude and the orientation of the magnetization and the relative rotations. In addition, we show, how relative rotations can induce gradients of density, concentration, or temperature perpendicular to the magnetization. In sect. 3.2 we discuss strains induced dynamically by temperature gradients, as well as reversible and irreversible heat or concentration currents induced by relative rotations.

\subsection{Static experiments}

There is a static coupling between elastic stresses and the orientation of the magnetization in eq. (20) provided by the material tensor $\chi_{i j k l}^{m}$ with two coefficients $\chi_{1,2}^{m}$ in eq. (23). Together with the rotational stiffness energy of the magnetization in eq. (7), given by the Frank-type tensor $K_{i j k l}$, eq. (8), and neglecting other cross-couplings, the stationarity condition, $\Psi_{i j}^{m}=0$, leads, for linear deviations from $\mathbf{m}=\mathbf{e}_{z}$, to

$$
\nabla_{z} m_{y}=A \varepsilon_{x z}^{0} \quad \text { and } \quad \nabla_{z} m_{x}=A \varepsilon_{y z}^{0}
$$

with $A=\left(\chi_{1}^{m}+\chi_{2}^{m}\right) \tilde{T}_{0} / K_{3}$. This describes an inhomogeneous rotation of the magnetization out of the shear plane of the external strain $\varepsilon_{x z}^{0}$ or $\varepsilon_{y z}^{0}$. This effect only occurs when tetrahedral order is present.

Similarly, a uniaxial compression along the preferred axis leads to linear deviations of the magnetization in the transverse plane given by

$$
\nabla_{x} m_{y}=\nabla_{y} m_{x}=\frac{\chi_{1}^{m}}{2 K_{4} \tilde{T}_{0}} \varepsilon_{z z}^{0} .
$$

This solution describes a spatial pattern, where the induced transverse magnetization is of constant magnitude $\left(m_{x}^{2}+m_{y}^{2}=\right.$ const) on circles around the $z$-axis, but changes its direction by $2 \pi$, when moving along the circle. This pattern has no splay, bend or twist character, but is quite special for tetrahedral order.

External strains $\left(\varepsilon_{x z}^{0}, \varepsilon_{y z}^{0}\right.$, and $\left.\varepsilon_{x x}^{0}-\varepsilon_{y y}^{0}\right)$ create spatial patterns of relative rotations, due to the $\chi_{1,2}^{\Omega}$ coupling in eq. (22), which, however, might be difficult to observe.
A well-known effect of external strains applied to ferromagnetic gels is magnetostriction, the change of the magnitude of the magnetization. Using the magnetostrictive coupling, described by $\gamma_{i j}$ in eq. (20), the homogeneous changes induced by compressional strains have the uniaxial form

$$
M_{0} \delta M=-\frac{\alpha}{\gamma_{\|}} \varepsilon_{z z}^{0}-\frac{\alpha}{\gamma_{\perp}}\left(\varepsilon_{x x}^{0}+\varepsilon_{y y}^{0}\right),
$$

where $\alpha$ is the magnetic stiffness coefficient.

In ferromagnetic gels with tetrahedral structure, in addition, external shear strains change the magnitude of the magnetization

$$
\alpha \delta M=-2 \tau_{\|} \tilde{T}_{0} \nabla_{z} \varepsilon_{x y}^{0}-2 \tau_{\perp} \tilde{T}_{0}\left(\nabla_{y} \varepsilon_{x z}^{0}+\nabla_{x} \varepsilon_{y z}^{0}\right)
$$

although the inhomogeneous external shear strains are probably not easy to apply.

Another possibility to probe magnetic and tetrahedral order is the application of a static relative rotation, $\tilde{O}_{i}$. Due to the coupling provided by $\psi_{i j}^{\lambda}$ in eq. (24) or (A.11), this results in gradients of the scalar variables $\lambda \in\{\sigma, \rho, c, M\}$ perpendicular to $m_{i}$ and $\tilde{O}_{i}$

$$
\begin{aligned}
& \nabla_{y} \lambda=\frac{D_{1}}{\psi^{\lambda} \tilde{T}_{0}} \tilde{O}_{x} \\
& \nabla_{x} \lambda=\frac{D_{1}}{\psi^{\lambda} \tilde{T}_{0}} \tilde{O}_{y}
\end{aligned}
$$

with $\psi^{\lambda}$ defined in eq. (25).

\subsection{Dynamic experiments}

As an example for a dynamic coupling, we discuss induced stresses due to an external temperature gradient perpendicular to the direction of the magnetization. Such couplings are specific for tetrahedral order. Already in the fluid case, there is a dissipative, $\Gamma_{i j k}^{(2)}$, and reversible, $\Gamma_{i j k}^{T}$, coupling according to eqs. (B.3) and (52), respectively,

$$
\sigma_{i j}=-\Gamma_{i j k}^{(2)} \nabla_{k} T-\Gamma_{i j k}^{T} \nabla_{k} T
$$

With the explicit form of the material tensors, eqs. (43) and (64), one gets for the temperature gradient applied along the $x$-axis, $\nabla_{x} T=\beta_{0}$ (and the magnetization along the $z$-axis) the induced stresses

$$
\begin{aligned}
\sigma_{x z} & =\sigma_{z x}=\beta_{0} \tilde{T}_{0}\left(\Gamma_{21}^{D}+\Gamma_{22}^{D}\right), \\
\sigma_{y z} & =\sigma_{z y}=\beta_{0} \tilde{T}_{0} \Gamma_{\perp}^{T},
\end{aligned}
$$

where the in-plane shear stresses are due to the dissipative coupling, while the shear stresses perpendicular to the temperature gradient result from the reversible coupling.

If the temperature gradient is along the $y$-axis, equivalent expressions for the appropriate induced stresses are found, with the same prefactor for the reversible coupling and opposite sign in the dissipative case. This reflects the 
breaking of transverse isotropy by the tetrahedral order. A temperature gradient along the magnetic field does not induce any stresses.

In a fluid phase such constant stresses only act as possible boundary conditions for flow and are difficult to measure. In a gel phase the stress tensor also comprises the elastic stresses, which are easier measurable by mechanical means. However, in the gel case there are additional couplings that effectively add to the response of stresses on external temperature gradients. In particular, temperature gradients induce non-zero values of $W_{i}$, the molecular field of relative rotations, due to the stationary condition $Y_{i}^{O}=0$. The couplings described by $p^{T}$ and $d^{W}$ in eqs. (B.8) and (57) are provided by the tetrahedral order. On the other hand, those induced non-zero values of $W_{i}$ act as forces that give rise to additional stresses via $\xi^{A}$ and $d^{A}$ in eqs. (B.3) and (52). As a result, induced elastic stresses $\Psi_{x z}=\Psi_{z x}$ and $\Psi_{y z}=\Psi_{z y}$ are obtained that are proportional to $\beta_{0} \tilde{T}_{0}$. The proportionality factors are lengthy expressions containing static susceptibilities and (in both cases) reversible and irreversible transport coefficients, which we will not show in detail here.

Applying a thermodynamic force $W_{i}$, by means of a relative rotation $W_{i}=D_{1} \tilde{O}_{i}$, eq. (A.11), heat and concentration currents, both reversibly, eqs. (50) and (51), as well as irreversibly, eqs. (B.1) and (B.2) are triggered of the form

$$
\begin{aligned}
& j_{x}^{(\sigma, c) R}=d^{(T, c)} \tilde{T}_{0} W_{x}, \\
& j_{y}^{(\sigma, c) R}=-d^{(T, c)} \tilde{T}_{0} W_{y},
\end{aligned}
$$

and

$$
\begin{aligned}
& j_{x}^{(\sigma, c) D}=p^{(T, c)} \tilde{T}_{0} W_{y}, \\
& j_{y}^{(\sigma, c) D}=p^{(T, c)} \tilde{T}_{0} W_{x},
\end{aligned}
$$

where $d^{(T, c)}$ is defined in eq. (60).

\section{On the influence of transient elasticity}

If the elastic network is not permanent, but transient, strains are relaxing (rather than diffusing). This means that elastic stresses $\Psi_{i j}$ act as thermodynamic forces (rather than $\nabla_{j} \Psi_{i j}$ ) and the dissipation function acquires additional contributions

$$
\begin{aligned}
R_{\text {relax }}= & \frac{1}{2} \tau_{i j k l}^{\Psi} \Psi_{i j} \Psi_{k l}+\xi^{T \Psi} T_{i j k} \Psi_{i j} \nabla_{k} T \\
& +\xi^{c \Psi} T_{i j k} \Psi_{i j} \nabla_{k} c+\xi^{M \Psi} T_{i j k} \Psi_{i j} \nabla_{k} h^{M} .
\end{aligned}
$$

The strain relaxation tensor $\tau_{i j k l}^{\Psi}$ has the same form as the viscosity tensor $\nu_{i j k l}$ in eq. (46) containing six relaxation times $\tau_{1}^{\Psi}-\tau_{6}^{\Psi}$. There are dissipative cross-couplings between the elastic stresses and temperature, concentration gradients and gradients of $M$. The form of the dissipative currents is given in appendix B.

The reversible currents have to fulfil the proper timereversal symmetry requirements and must not increase the entropy, i.e. they must fulfil eq. (39). Under that proviso we find

$$
\begin{aligned}
j_{i}^{\sigma R}= & \xi^{T R} T_{j k m} \epsilon_{i m l} m_{l} \Psi_{j k}, \\
j_{i}^{c R}= & \xi^{c R} T_{j k m} \epsilon_{i m l} m_{l} \Psi_{j k}, \\
X^{M R}= & \xi^{M R} \epsilon_{k l m} \nabla_{k}\left(m_{l} T_{i j k} \Psi_{i j}\right), \\
Y_{i j}^{R}= & \tau_{i j k l}^{R} \Psi_{k l}+\xi^{T R} T_{i j k} \epsilon_{k l m} m_{l} \nabla_{m} T \\
& +\xi^{c R} \epsilon_{k l m} m_{l} \nabla_{m} c+\xi^{M R} T_{i j k} \epsilon_{k l m} m_{l} \nabla_{m} h^{M},
\end{aligned}
$$

where the tensor $\tau_{i j k l}^{R}$ has the same form as $\nu_{i j k l}^{R}$ in eq. (61) with three parameters $\tau_{1}^{R}, \tau_{2}^{R}, \tau_{3}^{R}$. All the cross-couplings are possible due to the simultaneous presence of magnetic and tetrahedral order.

As far as static deformations are concerned, there is no difference between permanent and relaxing elasticity, and sect. 2.2 applies here as well.

If the elasticity is transient, one can induce elastic shear stresses directly using a temperature gradient. From eqs. (B.12) and (91) we get

$$
Y_{i j}=\left(\tau_{i j k l}^{\Psi}+\tau_{i j k l}^{R}\right) \Psi_{k l}+\left(\xi^{T R} T_{i j k} \epsilon_{k l m} m_{l}+\xi^{T \Psi} T_{i j m}\right) \nabla_{m} T .
$$

A stationary solution for the elastic stress is then obtained by setting all components $Y_{i j}=0$. These induced stresses are constant, and so are the additions to the heat current, eq. (B.9), preserving the stationarity of such solutions.

For a temperature gradient in the direction of the magnetization, shear stresses in the perpendicular plane are induced

$$
\Psi_{x y}=\frac{\tilde{T}_{0} \xi^{T \Psi} \nabla_{z} T}{2\left(\tau_{2}^{\Psi}+\tilde{T}_{0}^{2} \tau_{6}^{\Psi}\right)+8\left(\tau_{1}^{R}\right)^{2} / \tau_{2}^{\Psi}}
$$

involving dissipative as well as reversible transport parameters. The reversible couplings have also the effect of creating compressive stresses

$$
\Psi_{x x}=-\Psi_{y y}=-\frac{2 \tau_{1}^{R}}{\tau_{2}^{\Psi}} \Psi_{x y}, \quad \Psi_{z z}=0 .
$$

The result given in eq. (94) demonstrates the significant difference between reversible dynamic effects studied here and static magnetostriction presented in eq. (73).

If the external temperature gradient is perpendicular to the magnetization, it defines a preferred direction in this plane, which we will take without loss of generality as the $x$-axis, $\nabla_{x} T \equiv \Delta$. Then, of course, the $y$-direction is fixed by $\mathbf{m} \times \boldsymbol{\nabla} \mathbf{T}$. The orientation of the tetrahedron in the $x / y$-plane is arbitrary and the $x, y, z$ components of the four tetrahedral vectors can be written as

$$
T_{i j k}=\frac{T_{0}}{\sqrt{3}}\left(\begin{array}{cccc}
C+S & -C-S & C-S & -C+S \\
-C+S & C-S & C+S & -C-S \\
1 & 1 & -1 & -1
\end{array}\right)
$$

with $C=\cos \varphi$ and $S=\sin \varphi$. For $\varphi=0$ the projections of the four tetrahedral vectors are along the bisections $(1 / \sqrt{2})\left(\hat{\mathbf{e}}_{x} \pm \hat{\mathbf{e}}_{y}\right)$, while for $\varphi=\pi / 4$ they are along the $x-$ and $y$-axis, cf. fig. 1 . 


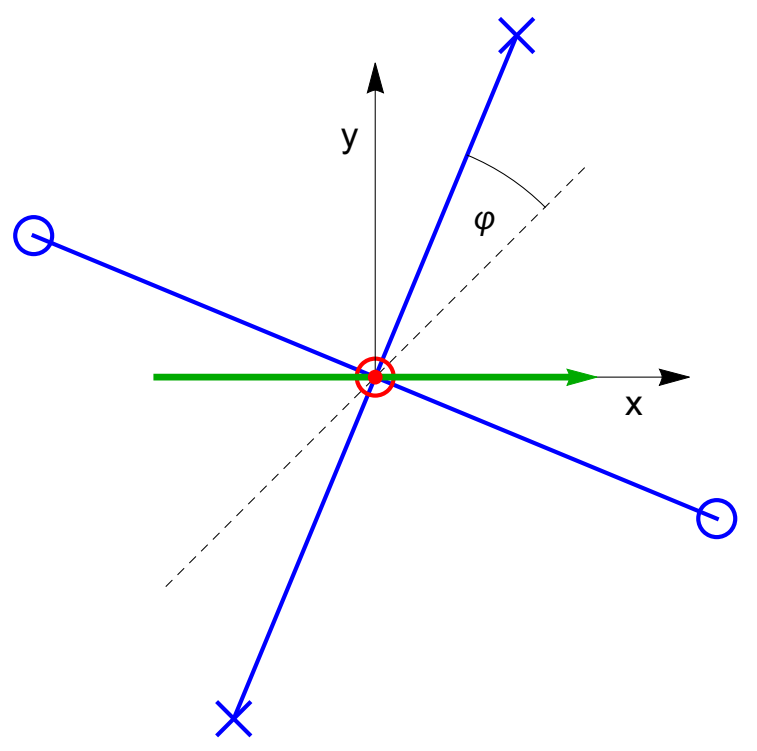

Fig. 1. The red circle in the middle indicates the magnetization perpendicular to the drawing plane, while the temperature gradient (green arrow) is along the $x$-axis. The blue lines show the projections of the tetrahedral vectors (where circles and the crosses represent vectors pointing out or into the plane, respectively).

Disregarding the reversible contributions in eq. (92) for the moment, we get the induced stresses

$$
\begin{aligned}
& \Psi_{x z}=-\frac{\xi^{T \Psi} \tilde{T}_{0} \Delta}{2 \tau_{5}^{\Psi}} \sin (2 \varphi), \\
& \Psi_{y z}=\frac{\xi^{T \Psi} \tilde{T}_{0} \Delta}{2 \tau_{5}^{\Psi}} \cos (2 \varphi) .
\end{aligned}
$$

As a result, the shear stresses are perpendicular to the temperature gradient for $\varphi=0$, while for $\varphi=\pi / 4$ the induced shear stress is in the $x / z$ plane given by the magnetization and the temperature gradient. In the general case, both types of stresses are present. By measuring $\Psi_{x z} / \Psi_{y z}$ one can determine the angle $\varphi$ and therefore the orientation of the tetrahedron. Alternatively, one could change the orientation of the temperature gradient to find one of the two special cases discussed above.

Taking into account also the reversible couplings, the results get more complicated, but the general features are similar

$$
\begin{aligned}
& \Psi_{x z}=\frac{A a-B b}{a^{2}+b^{2}} \tilde{T}_{0} \Delta, \\
& \Psi_{y z}=\frac{B a+A b}{a^{2}+b^{2}} \tilde{T}_{0} \Delta
\end{aligned}
$$

with

$$
\begin{aligned}
A & =-\xi^{T \Psi} \sin (2 \varphi)+\xi^{T R} \cos (2 \varphi), \\
B & =\xi^{T \Psi} \cos (2 \varphi)+\xi^{T R} \sin (2 \varphi), \\
a & =2 \tau_{5}^{\Psi}, \\
b & =2 \tau_{2}^{R}-2 \tau_{3}^{R} \tilde{T}_{0}^{2} .
\end{aligned}
$$

The orientation of the tetrahedral vectors, for which the resulting shear stress is perpendicular to the temperature gradient, i.e. $\Psi_{x z}=0$, is now given by

$$
\tan (2 \varphi)=-\frac{b \xi^{T \Psi}-a \xi^{T R}}{a \xi^{T \Psi}+b \xi^{T R}}
$$

The other special case, $\Psi_{y z}=0$, is still obtained by $\varphi \rightarrow$ $\varphi+\pi / 4$.

\section{Summary and perspective}

The macroscopic dynamics of ferromagnetic gels with tetrahedral order is rather peculiar due to two aspects. First, the permanent magnetization that spontaneously breaks part of rotational symmetry, is a variable that changes sign under time reversal. Second, the tetrahedral order, not only lifts the transverse isotropy perpendicular to the magnetization, but also breaks inversion symmetry already of the ground state. For the $D_{2 d}^{m g}$ phase, where the orientation of the magnetization is rigidly coupled to one of the tetrahedral $\overline{4}$ directions, we have discussed in detail, in the statics as well as in the reversible and irreversible dynamics, the possible cross-couplings among the three rotational symmetry variables, the strain tensor, relative rotations between the elastic network and the magnetization, and the usual fluid degrees of freedom.

We describe experimentally accessible effects that are specific for the $D_{2 d}^{m g}$ phase. In particular, we show that static external deformations lead to spatial patterns in the orientation of the magnetization. Shear deformations with the preferred direction in the shear plane trigger rotations of the magnetization direction out of the shear plane, while longitudinal compressions along the preferred direction result in a complicated, characteristic spatial pattern of the magnetization in the transverse plane. Both effects are only possible due to the tetrahedral order. In addition to the standard linear magnetostriction effects in ferromagnetic gels, the $D_{2 d}^{m g}$ phase also shows a kind of linear electrostriction, where the application of an electric field results in elastic shear stresses. Although there is no polarization present in the ground state of this phase, the necessary breaking of inversion symmetry is provided by the tetrahedral order. Another consequence of the tetrahedral order is a change of the magnitude of the magnetization due to external distortional deformation. Finally, relative rotations lead to gradients in the temperature, density, concentration, and the magnitude of the magnetization.

From the dynamics of tetrahedral fluids it is well known that, e.g. temperature gradients give rise to constant shear stresses. In a gel phase the stress tensor comprises also elastic stresses, which are easier measurable by mechanical means. In addition, in $D_{2 d}^{m g}$ there are additional couplings, mediated by relative rotations, that effectively add to the response of elastic stresses on external temperature gradients. Conversely, relative rotations trigger heat (and concentration) currents in the plane perpendicular to the magnetization. In the case of transient elasticity the elasticity is not permanent, but can relax 
either simply in the course of time (like polymers) or due to external (generalized) forces (eventually above a certain threshold value like in magneto-rheological systems). Here, the relaxation of strains can be compensated by e.g. external temperature gradients, such that stationary elastic stresses result. For gradients along the magnetization, elastic shear stresses, but also compressional ones (due to a specific reversible dynamic coupling), occur in the perpendicular plane. If the temperature gradient is perpendicular to the magnetization the resulting elastic shear stresses (in a plane that contains the magnetization direction) allow the identification of the transverse orientation of the tetrahedron relative to the gradient direction. This finding opens the door for the experiments investigating the orientation of the tetrahedron without the need of both magnetic and electric fields.

As a perspective it would be interesting to investigate the effects of dynamic (e.g., oscillatory) shear experiments on the orientation of the tetrahedral structure. Since several variables couple to the velocity field, both reversibly and dissipatively, one might expect a rich behavior depending on the amplitude as well as the frequency of the oscillations. Of special interest are also the rotations around the magnetization, which couple dynamically (neglecting the inhomogeneous stress forces) only to flow. It would also be important to investigate how the additional elastic network will influence the recently investigated tetrahedral ferromagnetic nematic liquid crystals.

Open Access funding provided by Max Planck Society. Partial support of this work by HRB, HP and TP through the Schwerpunkt programm SPP 1681 "Feldgesteuerte Partikel-MatrixWechselwirkungen: Erzeugung, skalenübergreifende Modellierung und Anwendung magnetischer Hybridmaterialien" of the Deutsche Forschungsgemeinschaft is gratefully acknowledged.

\section{Author contribution statement}

The authors have contributed equally to this paper.

Publisher's Note The EPJ Publishers remain neutral with regard to jurisdictional claims in published maps and institutional affiliations.

\section{Appendix A. Conjugate quantities}

For convenience we give the explicit form of the conjugate quantities that follow from the energy functional $f$, eq. (5), by partial derivation

$$
\begin{aligned}
v_{i} \equiv & \frac{\partial f}{\partial g_{i}}=\frac{1}{\rho} g_{i} \\
\delta T \equiv & \frac{\partial f}{\partial \sigma}=\frac{T}{C_{V}} \delta \sigma+\frac{1}{\rho \alpha_{s}} \delta \rho+\beta_{\sigma} \delta c-\nabla_{i}\left(\Pi_{i j k}^{\sigma} \nabla_{k} m_{j}\right. \\
& \left.+\Pi_{i j}^{\sigma M} \nabla_{j} M+C_{i j}^{\sigma \Omega} \nabla_{j} \Omega\right)+\chi_{i j}^{\sigma} \varepsilon_{i j}-\tau_{i j k}^{\sigma} \nabla_{k} \varepsilon_{i j} \\
& -\psi_{i j}^{\sigma} \nabla_{i} \tilde{O}_{j}
\end{aligned}
$$

$$
\begin{aligned}
& \delta \mu \equiv \frac{\partial f}{\partial \rho}=\frac{1}{\rho^{2} \kappa_{s}} \delta \rho+\frac{1}{\rho \alpha_{s}} \delta \sigma+\beta_{\rho} \delta c-\nabla_{i}\left(\Pi_{i j k}^{\rho} \nabla_{k} m_{j}\right. \\
& \left.+\Pi_{i j}^{\rho M} \nabla_{j} M+C_{i j}^{\mu \Omega} \nabla_{j} \Omega\right)+\chi_{i j}^{\rho} \varepsilon_{i j}-\tau_{i j k}^{\rho} \nabla_{k} \varepsilon_{i j} \\
& -\psi_{i j}^{\rho} \nabla_{i} \tilde{O}_{j} \\
& \delta \mu_{c} \equiv \frac{\partial f}{\partial c}=\gamma \delta c+\beta_{\sigma} \delta \sigma+\beta_{\rho} \delta \rho-\nabla_{i}\left(\Pi_{i j k}^{c} \nabla_{k} m_{j}\right. \\
& \left.+\Pi_{i j}^{c M} \nabla_{j} M+C_{i j}^{c \Omega} \nabla_{j} \Omega\right)+\chi_{i j}^{c} \varepsilon_{i j}-\tau_{i j k}^{c} \nabla_{k} \varepsilon_{i j} \\
& -\psi_{i j}^{c} \nabla_{i} \tilde{O}_{j} \\
& h^{M} \equiv \frac{\partial f}{\partial M}=-m_{i} H_{i}+\alpha M+\beta M^{3}-\gamma_{i j} M_{0} \varepsilon_{i j} \\
& -\tau_{i j k}^{M} \nabla_{k} \varepsilon_{i j}-\psi_{i j}^{M} \nabla_{i} \tilde{O}_{j} \\
& -\nabla_{i}\left(K_{i j}^{M} \nabla_{j} M+C_{i j}^{M \Omega} \nabla_{j} \Omega+K_{i j k}^{M m} \nabla_{j} m_{k}\right. \\
& \left.+\Pi_{i j}^{\sigma M} \nabla_{j} \sigma+\Pi_{i j}^{\rho M} \nabla_{j} \rho+\Pi_{i j}^{c M} \nabla_{j} c\right), \\
& h_{i}^{m^{\prime}} \equiv \frac{\partial f}{\partial m_{i}}=-M H_{i}, \\
& \Psi_{i j}^{m} \equiv \frac{\partial f}{\partial \nabla_{j} m_{i}}=K_{i j k l} \nabla_{l} m_{k}+C_{k i j}^{m \Omega} \nabla_{k} \Omega+K_{k j i}^{M m} \nabla_{k} M \\
& +\chi_{k l i j}^{m} \varepsilon_{k l}+\Pi_{k i j}^{\sigma} \nabla_{k} \sigma+\Pi_{k i j}^{\rho} \nabla_{k} \rho+\Pi_{k i j}^{c} \nabla_{k} c,(\mathrm{~A} .7) \\
& h^{\Omega^{\prime}} \equiv \frac{\partial f}{\partial \Omega}=0 \\
& \Psi_{i}^{\Omega} \equiv \frac{\partial f}{\partial \nabla_{i} \Omega}=K_{i j}^{\Omega} \nabla_{j} \Omega+C_{i j k}^{m \Omega} \nabla_{k} m_{j}+C_{i j}^{M \Omega} \nabla_{j} M \\
& +\chi_{k j i}^{\Omega} \varepsilon_{k j}+C_{i j}^{\sigma \Omega} \nabla_{j} \sigma+C_{i j}^{\mu \Omega} \nabla_{j} \mu+C_{i j}^{c \Omega} \nabla_{j} c, \\
& \Psi_{i j} \equiv \frac{\partial f}{\partial \varepsilon_{i j}}=c_{i j k l} \varepsilon_{k l}-\gamma_{i j} M_{0} \delta M+\chi_{i j}^{\sigma} \delta \sigma+\chi_{i j}^{\rho} \delta \rho \\
& +\chi_{i j}^{c} \delta c+\tau_{i j k}^{\sigma} \nabla_{k} \sigma+\tau_{i j k}^{c} \nabla_{k} c+\tau_{i j k}^{\rho} \nabla_{k} \rho+\tau_{i j k}^{M} \nabla_{k} M \\
& +\chi_{i j k}^{\Omega} \nabla_{k} \Omega+\chi_{i j k l}^{m} \nabla_{l} m_{k}+D_{2}\left(m_{i} \delta_{k j}^{\perp}+m_{j} \delta_{k i}^{\perp}\right) \tilde{O}_{k},
\end{aligned}
$$

$$
\begin{aligned}
W_{i} \equiv & \frac{\partial f}{\partial \tilde{O}_{i}}=D_{1} \tilde{O}_{i}+D_{2}\left(m_{j} \delta_{i k}^{\perp}+m_{k} \delta_{i j}^{\perp}\right) \varepsilon_{j k}+\psi_{j i}^{c} \nabla_{j} c \\
& +\psi_{j i}^{\rho} \nabla_{j} \rho+\psi_{j i}^{\sigma} \nabla_{j} \sigma+\psi_{j i}^{M} \nabla_{j} M .
\end{aligned}
$$

Since the $\delta$ 's in eqs. (A.2)-(A.4) describe deviations from the constant equilibrium values of the appropriate variable, all expressions on the left-hand side of the above equations are zero in equilibrium and can act as thermodynamic forces that drive the dynamics of the system. On the other hand, the right-hand sides of all these equations have to be zero in equilibrium (Euler-Lagrange conditions). Note that the energy $f_{\text {lin }}$ does not enter any Euler-Lagrange condition (except for $\nabla_{l} T_{i j k} \neq 0$ ), since it is linear in gradients of $m_{i}$.

\section{Appendix B. Dissipative currents}

For permanent elasticity the dissipative parts of the currents follow from the dissipation function $R$, eq. (40),

$$
j_{i}^{\sigma D} \equiv-\frac{\partial R}{\partial \nabla_{i} T}=-\kappa_{i j} \nabla_{j} T-D_{i j}^{T} \nabla_{j} \mu_{c}
$$




$$
\begin{aligned}
& -\psi^{T D} m_{j} T_{k j i} h_{k}^{m}-\Gamma_{k j i}^{(2)} A_{k j}-p^{T} T_{i j k} m_{k} W_{j} \\
& -\xi_{i j}^{T} \nabla_{k} \Psi_{j k}, \\
j_{i}^{c D} \equiv & -\frac{\partial R}{\partial \nabla_{i} \mu_{c}}=-D_{i j} \nabla_{j} \mu_{c}-D_{j i}^{T} \nabla_{j} T \\
& -\psi^{c D} m_{j} T_{k j i} h_{k}^{m}-\Gamma_{k j i}^{(3)} A_{k j}-p^{c} T_{i j k} m_{k} W_{j} \\
& -\xi_{i j}^{c} \nabla_{k} \Psi_{j k}, \\
\sigma_{i j}^{D} \equiv & -\frac{\partial R}{\partial A_{i j}}=-\nu_{i j k l}^{D} A_{k l}-c_{i j k}^{D} h_{k}^{m}-c_{i j}^{M} h^{M}-\tau_{i j} h^{\Omega} \\
& -\xi_{i j k}^{A} W_{k}-\Gamma_{i j k}^{(2)} \nabla_{k} T-\Gamma_{i j k}^{(3)} \nabla_{k} \mu_{c}-f_{i j k}^{D} \nabla_{l} \Psi_{k l}, \\
X_{i}^{m D} \equiv & \frac{\partial R}{\partial h_{i}^{m}}=b_{\perp}^{D} \delta_{i j}^{\perp} h_{j}^{m}+m_{j} T_{i j k}\left(\psi^{T D} \nabla_{k} T\right. \\
& \left.+\psi^{c D} \nabla_{k} \mu_{c}\right)+c_{j k i}^{D} A_{j k}+p^{m} T_{i j k} m_{k} \nabla_{l} \Psi_{j l} \\
& +\delta_{i j}^{\perp} \xi^{W m} W_{j}, \\
X^{M D} \equiv & \frac{\delta R}{\delta h^{M}}=b^{M} h^{M}+c_{i j}^{M} A_{i j}+\xi_{i j}^{M} \nabla_{i} \nabla_{k} \Psi_{j k} \\
& -p^{M} \nabla_{i}\left(T_{i j k} m_{k} W_{j}\right), \\
Z^{D} \equiv & \frac{\partial R}{\partial h^{\Omega}}=b^{\Omega} h^{\Omega}+\tau_{i j} A_{i j}, \\
F_{i}^{O D} \equiv & \frac{\partial R}{\partial \nabla_{k} \Psi_{i k}}=D_{i l}^{\Psi} \nabla_{k} \Psi_{l k}+\xi_{l i}^{T} \nabla_{l} T+\xi_{l i}^{c} \nabla_{l} \mu_{c} \\
& +f_{k j i}^{D} A_{j k}+T_{i l k} m_{k}\left(p^{W} W_{l}+p^{m} h_{l}^{m}\right) \\
& +\xi_{i j}^{M} \nabla_{j} h^{M}, \\
& +\xi_{i}^{W m} \delta_{i j}^{\perp} h_{j}^{m}+T_{i j k} m_{k}\left(p^{T} \nabla_{j} T+p^{c} \nabla_{j} c\right. \\
& \left.+p^{M} \nabla_{j} h^{M}\right),
\end{aligned}
$$

where $F_{i}^{D}$ is related to the dissipative strain current by $Y_{i j}^{D}=-\frac{1}{2}\left(\nabla_{j} F_{i}^{D}+\nabla_{i} F_{j}^{D}\right)$.

In case of a relaxing elasticity there are additional contributions in the dissipation function $R_{\text {relax }}$, eq. (87), giving rise to the following additional dissipative currents

$$
\begin{aligned}
j_{i}^{\sigma D} \equiv & -\frac{\partial R_{\text {relax }}}{\partial \nabla_{i} T}=-\xi^{T \psi} T_{k j i} \Psi_{k j}, \\
j_{i}^{c D} \equiv & -\frac{\partial R_{\text {relax }}}{\partial \nabla_{i} \mu_{c}}=-\xi^{c \psi} T_{k j i} \Psi_{k j}, \\
X^{M D} \equiv & -\nabla_{k} \frac{\partial R_{\text {relax }}}{\partial \nabla_{k} h^{M}}=-\xi^{M \Psi} \nabla_{k}\left(T_{i j k} \Psi_{i j}\right), \\
Y_{i j}^{D} \equiv & \frac{\partial R_{\text {relax }}}{\partial \Psi_{i j}}=\tau_{i j k l}^{\psi} \Psi_{k l}+\xi^{T \Psi} T_{i j k} \nabla_{k} T \\
& +\xi^{c \Psi} T_{i j k} \nabla_{k} c+\xi^{M \Psi} T_{i j k} \nabla_{k} h^{M}
\end{aligned}
$$

that come in addition to those of eqs. (B.1)-(B.8) shown above.

Open Access This is an open access article distributed under the terms of the Creative Commons Attribution
License (http://creativecommons.org/licenses/by/4.0), which permits unrestricted use, distribution, and reproduction in any medium, provided the original work is properly cited.

\section{References}

1. R.E. Rosensweig, Ferrohydrodynamics (Cambridge University Press, Cambridge, 1985).

2. S. Odenbach, J. Phys.: Condens. Matter 16, R1135 (2004).

3. H.-W. Müller, M. Liu, Phys. Rev. E 64, 061405 (2001).

4. B. Huke, M. Lücke, Rep. Prog. Phys. 67, 1731 (2004).

5. P. Ilg, E. Coquelle, S. Hess, J. Phys.: Condens. Matter 18, S2757 (2006).

6. V.I. Zadorozhnii, A.N. Vasilev, V.Yu. Reshetnyak, K.S. Thomas, T.J. Sluckin, Europhys. Lett. 73, 408 (2006).

7. S. Mahle, P. Ilg, M. Liu, Phys. Rev. E 77, 016305 (2008).

8. S.D. Peroukidis, S.H.L. Klapp, Phys. Rev. E 92, 010501 (2015).

9. M. Zrínyi, Trends Polym. Sci. 5, 280 (1997).

10. Z. Varga, J. Fehér, G. Filipcsei, M. Zrínyi, Macromol. Symp. 200, 93 (2003).

11. M. Zrínyi, L. Barsi, A. Büki, J. Chem. Phys. 104, 8750 (1996).

12. M. Zrínyi, F. Horkay, J. Intell. Mater. Syst. Struct. 4, 190 (1993).

13. M. Babincová, D. Leszczynska, P. Sourivong, P. Čičmanec, P. Babinec, J. Magn. \& Magn. Mater. 225, 109 (2001).

14. E. Jarkova, H. Pleiner, H.-W. Müller, H.R. Brand, Phys. Rev. E 68, 041706 (2003).

15. G. Pessot, P. Cremer, D.Y. Borin, S. Odenbach, H. Löwen, A.M. Menzel, J. Chem. Phys. 141, 124904 (2014).

16. S. Huang, G. Pessot, P. Cremer, R. Weeber, C. Holm, J. Nowak, S. Odenbach, A.M. Menzel, G.K. Auernhammer, Soft Matter 12, 228 (2016).

17. D. Collin, G.K. Auernhammer, O. Gavat, P. Martinoty, H.R. Brand, Macromol. Rapid Commun. 24, 737 (2003).

18. S. Bohlius, H.R. Brand, H. Pleiner, Phys. Rev. E 70, 061411 (2004).

19. L.G. Fel, Phys. Rev. E 52, 702 (1995).

20. L.G. Fel, Phys. Rev. E 52, 2692 (1995).

21. L. Radzihovsky, T.C. Lubensky, Europhys. Lett. 54, 206 (2001).

22. T.C. Lubensky, L. Radzihovsky, Phys. Rev. E 66, 031704 (2002).

23. B. Mettout, Phys. Rev. E 74, 041701 (2006).

24. L. Longa, G. Pajak, T. Wydro, Phys. Rev. E 79, 040701 (2009).

25. K. Trojanowski, G. Pajak, L. Longa, T. Wydro, Phys. Rev. E 86, 011704 (2012).

26. L. Longa, K. Trojanowski, Acta. Phys. Pol. B 44, 1201 (2013).

27. H.R. Brand, H. Pleiner, P.E. Cladis, Eur. Phys. J. E 7, 163 (2002).

28. P.E. Cladis, H. Pleiner, H.R. Brand, Eur. Phys. J. E 11, 283 (2003).

29. H.R. Brand, P.E. Cladis, H. Pleiner, Ferroelectrics 315, 165 (2005).

30. H.R. Brand, H. Pleiner, P.E. Cladis, Physica A 351, 189 (2005).

31. H. Pleiner, P.E. Cladis, H.R. Brand, Eur. Phys. J. E 20, 257 (2006).

32. H.R. Brand, H. Pleiner, Eur. Phys. J. E 31, 37 (2010). 
33. H. Pleiner, H.R. Brand, Eur. Phys. J. E 37, 11 (2014).

34. G. Pelzl, A. Eremin, S. Diele, H. Kresse, W. Weissflog, J. Mater. Chem. 12, 2591 (2002).

35. V. Bourny, V. Lorman, J. Pavel, B. Mettout, H.T. Nguyen, Ferroelectrics 276, 127 (2002).

36. W. Weissflog, M.W. Schröder, S. Diele, G. Pelzl, Adv. Mater. 15, 630 (2003).

37. T. Niori, J. Yamamoto, H. Yokoyama, Mol. Cryst. Liq. Cryst. 409, 475 (2004).

38. M.W. Schroeder, S. Diele, G. Pelzl, W. Weissflog, ChemPhysChem 2004, 99 (2004).

39. D. Wiant, J.T. Gleeson, N. Eber, K. Fodor-Csorba, A. Jakli, T. Toth-Katona, Phys. Rev. E 72, 041712 (2005).

40. J. Harden, B. Mbanga, N. Eber, K. Fodor-Csorba, S. Sprint, J.T. Gleeson, A. Jakli, Phys. Rev. Lett. 97, 157802 (2006).

41. D. Wiant, S. Stojadinovic, K. Neupane, S. Sharma, K. Fodor-Csorba, A. Jakli, J.T. Gleeson, S. Sprunt, Phys. Rev. E 73, 030703 (2006).

42. D. Wiant, K. Neupane, S. Sharma, J.T. Gleeson, S. Sprunt, A. Jakli, N. Pradhan, G. Iannacchione, Phys. Rev. E 77, 061701 (2008).

43. T. Ostapenko, D.B. Wiant, S.N. Sprunt, A. Jakli, J.T. Gleeson, Phys. Rev. Lett. 101, 247801 (2008).

44. Y. Jang, R. Balachandran, C. Keith, A. Lehmann, C. Tschierske, J.K. Vij, Soft Matter 8, 10479 (2012).

45. F. Vita, I.F. Placentino, C. Ferreo, G. Singh, E.T. Samulski, O. Francescangeli, Soft Matter 9, 6475 (2013).

46. M. Jasinski, D. Pociecha, H. Monobe, J. Szczytko, P. Kaszynski, J. Am. Chem. Soc. 136, 14658 (2014).
47. O.N. Kadkin, E.H. Kim, Y.J. Rha, S.Y. Kim, J. Taem, M.-G. Choi, Chem. Eur. J. 15, 10343 (2009).

48. E.H. Kim, O.N. Kadkin, S.Y. Kim, J. Taem, M.-G. Choi, Eur. J. Inorg. Chem. 2011, 2933 (2011).

49. H. Pleiner, H.R. Brand, Braz. J. Phys. 46, 565 (2016).

50. T. Potisk, H. Pleiner, H.R. Brand, Phys. Rev. E 98, 042703 (2018).

51. H.R. Brand, H. Pleiner, Eur. Phys. J. E 40, 34 (2017).

52. C. Dressel, T. Reppe, M. Prehm, M. Brautzsch, C. Tschierske, Nat. Chem. 6, 971 (2014).

53. C. Dressel, W. Weissflog, C. Tschierske, Chem. Commun. 51, 15850 (2015).

54. M. Alaasar, M. Prehm, Y. Cao, F. Liu, C. Tschierske, Angew. Chem. Int. Ed. 55, 312 (2016).

55. H. Temmen, H. Pleiner, M. Liu, H.R. Brand, Phys. Rev. Lett. 84, 3228 (2000).

56. H. Pleiner, M. Liu, H.R. Brand, Rheol. Acta 39, 560 (2000).

57. A.M. Menzel, H. Pleiner, H.R. Brand, J. Chem. Phys. 126, 234901 (2007).

58. A. Menzel, H. Pleiner, H.R. Brand, J. Appl. Phys. 105, 013503 (2009).

59. A. Menzel, H. Pleiner, H.R. Brand, Eur. Phys. J. E 30, 371 (2009).

60. H. Pleiner, H.R. Brand, Hydrodynamics and Electrohydrodynamics of Nematic Liquid Crystals, in Pattern Formation in Liquid Crystals, edited by A. Buka, L. Kramer (Springer, New York, 1996).

61. H.R. Brand, H. Pleiner, Eur. Phys. J. E 37, 122 (2014).

62. P.C. Martin, O. Parodi, P.S. Pershan, Phys. Rev. A 6, 2401 (1972). 